\title{
Moving pictures, moving on
}

6 There is richness at every street corner, under every roof', enthused $A C W$ 's reporter at 'Look at Britain!', the third Free Cinema programme, held at the National Film Theatre in mid-1957. ${ }^{1}$ Readers were urged to see how the profiled filmmakers linked with amateur practice and to emulate their challenge to re-observe the familiar: 'to look at it with honesty and affection ... relish its eccentricities, attack its abuses, love its people'. Encouraging amateur awareness of broader contemporary shifts in cinematic and visual practice recurred during the decades under review. Advocacy, in the hobby press, repeatedly positioned amateur activity in relation to professional cinema, as seen in published film criticism. Directors' profiles highlighted backgrounds in non-professional film production. Cinematic language, and the formalities of competitions, awards, festivals and other events that arose to shape the amateur calendar aligned some cine users with professional practice. Undoubtedly, amateur cinema became, for some of its exponents, visual practice unfettered by commercial considerations. Cine equipment and projection at home or in local club venues allowed freedom for personal expression and experimentation. Small-gauge film maximised scope for pursuing individual interests and available opportunities with limited resources. Undaunted by the pejorative tags and jibes aimed in their direction by doubters, some amateur enthusiasts pursued their activity with dedication. Sustained by shared interests and the companionship of fellow practitioners, their sometimes lifelong commitment gave rise both individually and collectively to considerable artistry, talent and endeavour as brought to new audiences by an emerging number of scholars in recent years.

At the other end of the amateur spectrum were numerous do-it-yourself cine-equipment users, whose commitment, skills, resources and motives varied. Understanding Britain's amateur filmmaking movement as a continuum 
stretching between casual usage and high-quality cinematography that reflects expertise and many years of experience helps to accommodate this vast range of output that exists within public archives and still in private hands. Finding ways to understand this disparate body of work that neither relegate footage as visually naive and banal nor bubble-wrap the aesthetically sophisticated production in narrow theoretical concepts lies at the heart of the present text. Writing has striven not to alienate the very constituency whose enthusiasms have sustained amateur activity against decades of rapid technological development and societal change. Some of those very practitioners, indeed, have contributed to the present project. Crafting a text informed by contemporary concerns and scholarship whilst ideally remaining faithful to the intentions and integrity of successive generations of amateur practitioners has been important.

Undeniably, amateurs and their audiences found something intensely compelling in the cine-camera's capacity to record moments of being there. Reliving particular events or the ability to join in as armchair travellers prompted part of the interest and delight. The shared experience of making and watching amateur films together, particularly beyond the family circle, but rather with friends, club members and local people, was not merely a 'social good', in sociological terms - a process of bringing people together. Its reassuring promise of belonging and shared involvement in affective pleasures created an intimate and empowering semi-public sphere that perhaps assuaged wider uncertainties of societal and political change, not unlike more contemporary interests in shared reading and choral singing.

For some people, the medium's visual pleasure existed precisely in recalling the transient enjoyments of the ordinary - the visual ephemeralities of the past, with the help of either known or unfamiliar filmmakers. Conversely, for others, the handheld camera and amateur's informal point of view, however compromised by poor quality, lighting or technical prowess, beckoned as invitations to step imaginatively into other worlds. Take, for example, the praise in $A C W$ following the public screening in late 1952 of the explorer-anthropologist Thor Heyerdahl's epic Kon Tiki voyage across the Pacific on a papyrus raft. ${ }^{2}$ There was jerky camerawork shot at sea level, scratched film emulsion, overly rapid panning, rusted film cans and water damage, and unmatched shots and sequences. Yet, the six crew members who used $16 \mathrm{~mm}$ film for the first time - later blown up to $35 \mathrm{~mm}$ for public screening - were commended for their 'triumph of teamwork', inspiration and capturing of 'remarkable thrills'. Amateur film, whether on the garden lawn, on the school playing field or in 
more exotic locations, invited its makers and audiences to sidestep time and, for the duration of the film, to inhabit alternative realities of various kinds. Produced alone or with others, amateur material stands as testimony to how people spent their time, sometimes engaged in 'serious leisure' and sometimes simply indulging a passing fancy for an activity that became available. ${ }^{3}$

Highlighting some of the scope and variety of amateur practice through offering a thematic lens and regional focus that, at times, has drawn up relevant material elsewhere, has been central to this project. No single area can encapsulate the variety of British or even English regional experience, but the diversities found within the main study location and periodic wandering across its boundaries to adjacent rural, industrial and urban localities offer a sufficiently coherent provincial focus suitable for future comparisons with other regions and metropolitan areas. The preceding discussion has drawn on virtually no material from Wales, Scotland or Northern Ireland, with the exception of the Scottish Amateur Film Festival. Claims that a regional focus offers a means for exploring a national movement seem valid, however, in that amateur practice has been related to aspects of national socio-historical, cultural and economic experience and has drawn upon the most continuously influential publications in Britain's advisory literature. A different regional setting, would inevitably skew the content differently but underlying the cited examples run themes, concerns and perspectives that merit a broader positioning of material, largely drawn from the nation's largest public regional film archive outside London, as a means to understand fifty years of twentieth-century British experience through amateur eyes.

Inevitably, the task has grown beyond its initial remit. Since its inception, developments in different areas of scholarship have been germane to the writing. Awareness and interest in amateur film has grown too, creating a shifting background for the present work. Educators, visual artists, filmmakers, broadcasters and other users have acknowledged the significance of amateur film footage in unprecedented ways. Archivists and collectors have become increasingly visible at the forefront of innovative partnerships that use amateur footage as part of broadening access to visual culture. Arts and heritage organisations have seen the potential popular appeal of flickering archive image on monitor screens even as the rise of multiple channels has precipitated a rash of independent producers searching for the unexpected, unmediated personal testimony and quirkiness believed to attract audiences.

Such activity is occurring against a background of shifting priorities in public sector spending and looming reductions to the funding of arts, culture, 
teaching and research. The explosion of digital technologies and their impact on personal image-making and the electronic implications for storing, accessing and distributing archive amateur materials pose challenges and opportunities that seem too important to ignore. Acknowledging the implications of such dynamic thrusts and turns within - and, as importantly, beyond academic enquiry, highlights the value underlying the interdisciplinarity and multiple standpoints that are now associated with understanding amateur film. Some thoughts on future directions in amateur film scholarship seem a fitting conclusion. If not an agenda, as scope for further study of different kinds has been identified in relevant sections already, the following comments may be seen as a positioning of this study in the emerging historiography and intellectual mapping out of amateur cinema studies.

Growing academic interest in amateur activity has broadened theoretical and methodological approaches. Exploration of surviving amateur footage now ranges across themes, formats, genres - including fiction, animation, historical, colonial, travelogue and documentary - and its textual and cultural practices. Yet, for some scholars, its relationships with other forms of moving image - and photography too - remain prime considerations. As the complex interplay of past links between amateur and professional practice is gaining recognition through emerging studies of individual filmmakers, or the role of support organisations, clubs, festivals, competitions and specialist literature, fresh attention is drawn to more theoretical concerns about how amateur films are talked and written about now and in the past.

For decades, Britain's specialist press reverberated with references to current films and terminologies used in film criticism in ways that were both eclectic and enthusiastic, as seen early on in Macpherson's idiosyncratic Close Up. While this short-lived magazine embodies how some writing about different cinematic forms was inflected with prevailing concerns about modernism, art, literature and society, much amateur activity occurred away from elite discursive practice, even if not totally isolated from wider trends in the viewing and screening of moving imagery. Other amateur publications had more middle-brow appeal in their mix of advice and criticism and yet, for some cine users, even these instructive tones were not part of how they practised their hobby. Indeed, commentators sometimes lamented the apparent gulf between critical awareness honed by actually going to the cinema and much amateur output. Issues of intentionality seem valid here, in remembering the breadth of amateur visual practice and the appropriateness of transferring critical perspectives between contexts. Even within later serial publications, contributions 
ensured different voices and a plurality of perspectives that would reach a wide readership.

As with any language, evolution occurs as expressions borrow, import, absorb and graft from different sources and give rise to new ways of communication. While some amateurs used art-house and mainstream film-related lexicons to strengthen the status of amateur cinematography - perhaps enhancing their own position too - it seems likely that language of film - and later television - also gained some popular acceptance too, as borne out by dipping into dictionaries and other verbal texts of the time. An aesthetic history of amateur practice could further develop the trajectories of these co-existent discursive forms within amateur settings. Contemporary approaches to amateur film may likewise gain from applying the critical vocabularies and constructs of film studies. Such steps might offer analytical lenses that assist with its repatriation within film studies, and have legitimacy among those wishing to further links with creative practitioners and scholars from film, media and visual backgrounds.

Adopting the language of contemporary film studies in the analysis of amateur practice may also help in repositioning the latter within more inclusive cinematic histories. Since the study of emerging national cinema remains an important element of twentieth-century film history, where amateur activity predates production at a national level, might amateur film be considered as an underacknowledged fledging step in the development of a country's cinema? Valuable contributions are being made by researchers who examine amateur film practice within specific national contexts, rather than conceiving it as an undifferentiated trans-national hobby contingent upon international marketing, publishing and organisational structures. Comparative studies on amateur activity will further our understanding of both content and context.

Connecting with other relevant areas of film-related study and, almost as importantly, regional television histories from the 1950s onwards need not eclipse the potential for other interpretative frameworks. Memory studies is one such burgeoning area of enquiry where concepts and approaches contribute to understanding processes underlying the making, sharing, and passing on of private and public visual memories. While digital technologies impact upon processes of remembering and forgetting, research into psychoanalytical, neurocognitive and biological systems broadens established socio-cultural, literary and artistic areas of interest in acts of recollection, forgetting, avoidance and erasure. ${ }^{4}$ Long-established metaphors of memory as storehouse, archive and other forms of repository have undergone reappraisal as new vocabularies, 
springing from the previously hidden survivor narratives of conflict, disaster, trauma and genocide, have gained recognition. ${ }^{5}$ This paradigm shift opens ways of understanding memory as being more fluid, dynamic and complex than hitherto implied by the fixity implicit in earlier cultural imagery. ${ }^{6}$ For those working on questions of visual memory and identity, more transformative approaches to making sense of amateur material have become available.

Amateur film, and its video and digital successors, evolved against decades that witnessed total war, prospects of annihilation and the powerful role of human agency in creating landscapes of dereliction and ruin. As the capacity for obliterating traces of the past soared, mimetic means to record expanded from the early brief fragments of exposed film less than a minute long to the life-logging possibilities that digital media now permit. Exposing this mismatch between the recordable and the recording process highlights more philosophical concerns over the ultimately unknowable and incomprehensible nature of times past. Couching the significance of past amateur footage in terms of its evidential value as visual history continues to play an important role in establishing and furthering amateur film scholarship but more nuanced interpretations of memory-making offer exciting possibilities to the study of amateur materials at a point where the absences, gaps and discontinuities in recollection, and in time itself, seem to converge.

Dynamic notions of memory-making and shaping, like more multi-layered ideas about identity formation, selfhood and belonging, have greater relevance for some areas of amateur film than others. Both have seemed pertinent to the discussion of material broadly conceived within a realist tradition as considered in these pages. Such concepts seem least applicable to amateur animation, other than, perhaps, in oral histories of individual practitioners. While finding points of commonality may appear to undermine the breadth of amateur practice, it identifies scope for less obvious points of investigation, as in details of past social practice and gender roles that exist in the relatively small number of amateur fiction productions currently held in public archives and better represented within club collections. More generally, visual clues to past lives and landscapes offer amateur footage a focus on visibility and materiality that appears at odds with the elusive flickering nature of its projected image and beam of light. While the insubstantial nature of the image - as opposed to the mechanical presence, form and sound of cine equipment - distances it from the materiality of a photograph and the sensory encounter of holding an album, amateur film's frequent visual focus on people, places and objects creates an unlikely alliance with recent conceptual shifts in archaeology.? 
Long seen as the poor relation to history, archaeology developed in part outside the frameworks and dialogues of its supposedly more intellectually significant and text-based superior. It offers an interesting parallel to the erstwhile marginalisation of amateur activity within broader cinematic study. Whilst being analogous to repositioning amateur film scholarship within discussions on film and cinema history, this comparison highlights the enriching insights available from elsewhere. Both subjects share an interest in fragments from the past and acknowledge the inherently selective process of making and leaving traces in time. If excavation has dealt with the discarded rubbish of the past, amateur activity, particularly in earlier dismissive notions about home movies, was viewed with similar contempt. Attempts to reinstate two once marginalised activities within more inclusive dialogues offer points of similarity.

Both fields of study share concerns with what survives and the problematic gaps in what is left unsaid, unseen and remains unrecoverable. ${ }^{8}$ The omissions, absences, discontinuities and unseen presences are starting points for investigations that seek to step beyond the frame and widen the search for meaning. Issues of provenance, relocations, and agency and action, are of mutual importance, as shown by how archival discoveries, like those unearthed from the ground, gain fresh meanings when seen and shared in different settings. Ethical issues may arise as shifts occur from once private, or neglected and forgotten places to more public spheres. Intentionality, purpose, function, reception and value may be as elusive for the excavated artefact as for an amateur cine reel. Materiality, and chance survival, may confer on both a longevity that outlasts their original makers and users. They may even acquire their own narratives, memories and new significance.

Making sense of multiple stories and alternative perspectives has given rise to notions of multi-vocality within archaeology and cultural anthropology. It offers a potential framework for accommodating the variety of past experiences found within amateur material too, particularly when dealing with the abundance of similar content within personal and family film recording. Multi-vocality offers a multi-stranded alternative to the constructing of historical memory-making. Different visual versions may be understood alongside each other as complementary traces of the past. Archived private footage is not simply the unofficial version of past experience made visible; it represents repeated instances of individual expression on a large scale. So, birthdays, beach scenes, picnics and tea parties, for instance, simply through their replication over time by different amateurs, gain value that extends beyond their individual worth. 
Family stories, whether in words or pictures, communicate notions of self and situation and link past, present and future. Although an analogy with oral traditions can not be taken very far since footage lacks the malleability and longevity of being passed down by storytellers and their audiences, it does point to the importance of negotiated spaces in which presenter and audience may share in making disparate 'texts' meaningful. If excavated artefacts acquire significance through uncovering their wider setting, then the stories told on amateur film - just like oral traditions - gain from being related to their social, political and historical contexts. Both expose the subjective and sometimes provocative nature of interpretation, and are reminders of how the past is contested as it prompts fresh interests in contemporary social and political settings.

During decades when the acceleration of time generated new ways of experiencing, conceptualising, dominating and representing the world, amateur filmmakers explored their own ways to manipulate time, challenging and reworking logics of sequence and linearity. Archaeology likewise moved beyond narrow sequential framing of stratified layers to comparative approaches, entering into new dialogue with cultural anthropology, contemporary art, design and comparative indigenous studies, to bring new explanations and insights. Amateur footage similarly benefits as scholars, creative practitioners and varied audiences expose, juxtapose, locate and encounter its imagery differently. Meanings evolve and significances change as shown in the preceding chapters. Finding effective languages permits different kinds of conversation about past visual pursuits. Aesthetics, techniques, sounds, silences, intertitles and story boards may be understood afresh as amateur film scholarship engages with other areas of thought and practice.

The archaeological metaphor may be taken further by those who excavate media prehistory to recover the layers and genealogies of past practices and technologies of communication. Linking the record-making impulse between home movies and mobile phone technologies traces a desire to see and share technically from modernity to postmodernity and propels amateur film practice beyond a cinematic framework into narratives of communication history. Early cine practices legitimately nestle within more inclusive and unconventional histories of communication that embrace filmic histories as media archaeology. ${ }^{10}$ Work on the role of home video technologies in the transition from cine film to digital image-making augments this evolutionary route still further. The 'hiddenscapes' of amateur visual activity - those patterns of meanings and details below the surface, invisible without careful investigation, await uncovering: imagery, like landscape, is inscribed with past ideas, 
assumptions and values that also inform contemporary sensibilities.

In the digital era, Witmore suggests, memory has become cheap. ${ }^{11}$ Film length, development costs and album accumulation long constrained the taking of analogue photographs. Moving imagery was likewise limited by resources until video technologies arrived. New media's capacity for personal image-making moved from hesitancy to profligacy in under two decades, generating an explosion of visual mementoes, as well as an unprecedented facility to store, show and share picture memories. If digital technologies have impacted upon visual memory-making, they have also recast amateur film and video practice as direct visual antecedents to the mimetic processes of today. The British launch of Pathé and Kodak's camera and projector equipment for the home market in the early 1920s established precursors to contemporary media technologies. This fifty-year survey of amateur film may itself be seen as an archaeological intervention in mapping out Britain's personal image making practices and cultures over the past nine decades. While study of digital memories is evolving fast, the comparative perspectives offered by amateur activity help to position the digital turn within a wider understanding of visual memory studies. Grappling with the impact of digitisation upon memory at personal, public and collective levels may be new but the mental, emotional, social, cultural and practical consequences of technological shifts in mediating memories have happened before with the advent of printing and in relation to photographic and media developments. Arguably, different evolving communication technologies have each affected the human capacity to remember, so present-day technologies of the self, to borrow Foucault's phrase, are but the latest in a succession of ways to redefine the boundaries, conventions and constructions of autobiographical and collective remembering and forgetting.

This longer perspective informs comparison of past and present visual practices. Given current capabilities in digital capture of everyday visual flotsam, earlier dismissive treatment of amateur activity as parochial and limited in content now seems misleading. Far from simply recording the routines of daily life, the selective nature of past cine use appears striking when set alongside the infinite nature of today's digital self-recording pursuits. Using a cine-camera was a conscious but, for some amateurs, highly sporadic intervention, often prompted by an occasion being special for a particular reason. The resultant visual record may seem trivial in some of what it shows, but what promoted the moments of shooting had significance. Visual fragments of times past, precisely because of their capacity to show again, now acquire value simply for their distance from the present. 
As instances of outreach to different communities in widely different contexts reveal, historical visual fragments are not merely animated versions of local history. Their gaze upon specific situations and experiences, which have been transformed or no longer survive, may help to offset patterns of myopia, erasure and severance that exist from domestic to global level. In different settings, working with amateur film and its successor video technologies, including those often problematic sequences shot by privileged outsiders, now offer the potential to help reconnect generations, overcome alienation and build bridges across physical, social, and psychic chasms. Virtual visual repatriation may involve no more than posting an archive clip and perhaps monitoring responses but its online presence may be a catalyst for gathering other evidence and renewed cultural visibility. ${ }^{12}$ The challenges of such work are considerable while public funding for such schemes that seek to recover lost knowledge and rekindle hope tends to be limited. ${ }^{13}$ From the Arctic to sub-Saharan Africa, there is scope for taking amateur visual imagery out of the archive, whether as part of widening access to collections or sensitive schemes of visual repatriation, knowledge-sharing or community empowerment. Far from being nostalgic indulgence, such ventures may enhance understanding within and between different peoples. Will family films in cine or video format once shot in and around the comfortable homes of Belfast, Bagdhad, Bulawayo or Bloomfontein - currently discarded, unloved or despised - one day have future roles and relevance in helping people come to understand their pasts and presents differently? ${ }^{14}$ Can footage of communities and localities marginalised and divided by race, religion and reputation find alternative ways of understanding self, circumstance and sense of direction, though engaging with the visual snippets of earlier times? ${ }^{35}$ Certainly, recent and ongoing initiatives offer some cautious encouragement as evolving technologies bring old formats to new audiences and venues.

While accessing digital and cine or video memories relies upon other equipment, digitisation has expanded image availability, far beyond the original personal sphere of domestic or semi-public club space. Scale poses its own challenges: individual storage of personal material was hard enough as cine reels and cassettes accumulated over time, with or without titles and re-editing. Digitisation throws open the once private archive of stored visual memories in new ways: imagery is shared, circulated and remixed even as some individual items are lost in the process. Beyond the challenges of organising data and its retrieval on a personal computer exist global possibilities of accessing data, as unknown amounts of material are streamed and made available online. The 
practical, ethical and conceptual consequences of such electronic abundance carry risks and rewards.

Opening archival access breathes fresh life and possibilities, and generates creativity, interest and new audiences for working with archive footage, in and beyond academic circles. Such strategies within archival practice highlight new emphasis upon visibility, outreach and self-justification, and overturn traditional roles as keepers and protectors of archive materials. Putting material online means it enters a global public domain. Inevitably, the online presence of archive amateur footage is skewed by resources, so that some repositories are more able to digitise than others. Changing patterns of availability emerge in new configurations of unevenness at regional, national and international levels. Whilst not forgetting the digital divide and those that lack access for different reasons at national and international level, digitisation enables wider access to moving imagery of all kinds from social networks too, as shown by the upsurge of archive footage on YouTube and equivalent sites since its inception. Arguably, archives have a greater longevity than YouTube and its equivalents, even in the present economic landscape.

How vulnerable could past amateur footage become through digital access? The extent of digitisation, availability and usage are as yet unknown. Amateur content and aesthetic is appealing to different users, but online availability should not make it any more exploitable than professionally made archive film. Often erroneously assumed to be freely available without seeking copyright permission from its owners or its archival home, streaming amateur material requires the same clearance by the archival host as needed by any user approaching via conventional routes. Although streaming offers greater visibility and easier access rather than making a quantitative change, in practical terms it seems to make more material available. The potential visual pick and mix available through electronic access seems to pose specific threats to amateur material but those risks relate in part to how data is digitised and appears on line, with or without contextual details. The integrity of imagery, and how it is found and reworked by users, forms part of an evolving agenda of online archival practice.

More and more collectors and archives, internationally, feel that open access ultimately raises awareness and contributes new significance as imagery gains fresh meaning in different contexts. Within what some might see as an emerging era of global visual commons, new notions of regularity practice have yet to gain consensus, let alone adoption and usage. Visual artists, archivists and scholars are still coming to terms with the sheer freedom of possibilities as 
hitherto unavailable materials become accessible. Issues of ethics, integrity and even the very basics of contextual research to establish provenance, intentionality and other details of origin rely upon the time, commitment and skills of individual image-searchers. Among those using, working with and responsible for archive footage, exemplary research and creative practice urgently deserves more publicity so that new interpretations and approaches do not take place in a critical or visual vacuum or sever amateur material from its wider narratives.

As digital scholarship offers alternative tools within the arts and humanities for collaborative ways to generate and interpret materials exchanged via digital means - with implications for teaching and learning, scholarship, knowledge creation and dissemination, and participation through virtual means - archive visual material acquires new potency. Archive amateur imagery is a means to question how people constructed ways of knowing about themselves, their families and friends, and others in the world. It is a means to reflect upon successive visual acts and interventions that constitute cultural memory and experience. Delving into the stories and meanings accompanying the amateur films considered in this book offers a reminder of the richness that those perspectives may also bring. As amateur film practice, historiography and scholarship approach their centenary, the delights and passions underlying its visual pursuits seem integral to understanding past amateur activity. Conversations about amateur film practices - in all senses - are entering a fascinating phase. This study seeks to be one step towards greater understanding.

\section{Notes}

1 (Anon.), 'They call it free cinema'.

2 T. Heyerdahl, 'How I filmed Kon Tiki', 780-1, 786; L. Wood, 'At your cinema remarkable thrills'.

3 I. Craven, Movies on Home Ground.

4 A. Erll and A. Nunning, Memory Studies.

5 J. Brockmeier, 'After the archive: remapping memory'.

6 S. Nalbantian, Memory in Literature: from Rousseau to Neuroscience.

7 See for instance, I. Hodder (ed.), Archaeological Theory Today.

8 A. Gonzalez-Ruibal, “The Dark Abyss of Time”, accessed on 10 September 2010.

9 I. Hodder, Symbols in Action.

10 Elsaesser, T., 'The new film history as media archaeology'.

11 C. Witmore, 'Between media archaeology and memory practices: two recent excavations', accessed on 10 September 2010.

12 Conversation with Pam Wintle, senior archivist at Human Studies Film Archives, Smithsonian Institution, Washington DC. about streaming footage of Bamiyan Valley, 23 September 2010. 
13 Caroline Forcier Holloway (Library and Archives Canada, Ottawa) kindly drew my attention to Project Naming, www.collectionscanada.gc.ca/inuit/index.html. Outreach to northern communities has used archive still imagery to foster intergenerational knowledge and memories-sharing and archive footage during the tenth anniversary of Nunavut (January 2009), Personal communication with author, 27 July 2010.

14 Passing reference to home movies during an interview by Gabriel Gatehouse, 'Iraq's growing disquiet at lack of infrastructure' in BBC Radio 4, 'From Our Own Correspondent', 3 July 2010.

15 H. Norris Nicholson, 'Moving pictures; moving memories: framing the interpretative gaze'; 'Manchester's Moving Memories: Tales from Moss Side and Hulme: archive film and community history-making' (2012). 